\section{THU0532 PAEDIATRIC UVEITIS IN FRENCH REFERRAL OPHTHALMOLOGIC CENTERS: A DESCRIPTIVE ANALYSIS OF 74 CHILDREN}

L. Moal ${ }^{1}$, A. Rousseau ${ }^{2}$, C. Titah $^{3}$, M. Labetoulle ${ }^{4}$, B. Bodaghi ${ }^{5}$, S. Guillaume Czitrom ${ }^{6} .{ }^{1}$ Service de Medecine des Adolescents; ${ }^{2}$ Service d'Ophtalmologie, $\mathrm{CH} \cup$ Bicetre, le Kremlin Bicetre Cedex; ${ }^{3}$ Service d'Ophtalmologie, Fondation Rothschild, Paris; ${ }^{4}$ Service d'Ophtalmologie, $\mathrm{CHU}$ Bicetre, le Kremlin Bicetre; ${ }^{5}$ Service d'Ophtalmologie, $\mathrm{CHU}$ Pitie-Salpetriere, APHP, Paris; ${ }^{6}$ Service de Medecine des Adolescents, CHU Bicetre, APHP, le Kremlin Bicetre, France

Background: Uveitis in children is rare. Intensive interactions between ophthalmologists and paediatric rheumatologists are needed in order to choose the best therapeutic strategies for severe uveitis attacks.

Objectives: Describe a cohort of 74 patients with paediatric uveitis.

Methods: Retrospective analysis of children followed for uveitis before 18 , by one paediatric rheumatologist (SGC) for systemic treatments' management and members of 3 ophthalmologic departments specialized in uveitis care in children (AR, CT, ML and BB) in Paris, during the 2006-16 period.

Results: There were 74 paediatric uveitis, 42 anterior (57\%, group 1$), 16$ intermediate $(21 \%$, gr2), 7 posterior $(9 \%$, gr3) and 9 pan-uveitis $(12 \%$, gr4 $)$. Gender was equal in gr2-4, but there were more females in gr1. At presentation, mean ages were $8.6 \pm 4.1,9.8 \pm 3.9,9.1 \pm 3.6$ and $10 \pm 4.2$ years old. Mean follow-up was $3.7 \pm 3.7$ years. JIA was the leading cause of gr1 uveitis (45\%); gr2-3 uveitis were idiopathic in $81 \%$ and $86 \%$, respectively. In gr4, etiologies were found in 7 out of 9 patients (Behçet-3, JIA-2, BBS-1, TINU-1).

Table 1

\begin{tabular}{lcccc}
\hline Complications & $\begin{array}{c}\text { I: Anterior } \\
\text { uveitis (42) } \\
51 \%\end{array}$ & $\begin{array}{c}\text { II: Intermediate } \\
\text { uveitis (16) } \\
71 \%\end{array}$ & $\begin{array}{c}\text { III: Posterior } \\
\text { uveitis (7) } \\
71 \%\end{array}$ & $\begin{array}{c}\text { IV: Panuveitis } \\
(9)\end{array}$ \\
\hline Cataracts & $25 \%$ & $21 \%$ & & $100 \%$ \\
Papilledema & $19 \%$ & $21 \%$ & & $44 \%$ \\
HTP/Glaucoma & $16 \%$ & & $29 \%$ & $56 \%$ \\
Macular edema & & $21 \%$ & & $33 \%$ \\
Vitreous hemorr. & & $29 \%$ & & $33 \%$ \\
Retinal detach. & $6 \%(n=2$ Uni) & $7 \%(n=1$ Uni) & $29 \%(n=2 B i)$ & $33 \%(n=2$ Uni+1Bi) \\
Blindness & & & &
\end{tabular}

Conclusions: Paediatric uveitis induce a very high-level burden in children, even when anterior and sometimes despite optimal therapeutic management in tertiary care centers. Their early recognition and tight control in specialized units are absolutely required in order to decrease the level of definitive complications. Disclosure of Interest: None declared

DOI: 10.1136/annrheumdis-2017-eular.6629

\section{THU0533 DO JIA CORE OUTCOME VARIABLES AT BASELINE PREDICT CLINICALLY INACTIVE DISEASE STATES AT ONE YEAR?}

S.J.W. Shoop-Worrall $^{1,2}{ }^{1}$, S.M. Verstappen ${ }^{3}$, J.E. McDonagh ${ }^{4,5,6}$,

W. Thomson ${ }^{4,7}$, K.L. Hyrich ${ }^{3,4}$ on behalf of CAPS. ${ }^{1}$ Arthritis Research UK Centre for Epidemiology, the University of Manchester; ${ }^{2}$ NIHR Manchester

Musculoskeletal BRU, Central Manchester University Hospitals NHS Foundation Trust and University of Manchester Partnership; ${ }^{3}$ Arthritis Research UK Centre for Epidemiology, the University of Manchester; ${ }^{4}$ NIHR Manchester

Musculoskeletal BRU, Central Manchester University Hospitals NHS Foundation Trust and University of Manchester Partnership; ${ }^{5}$ Centre for MSK Research, the University of Manchester; ${ }^{6}$ Manchester Academic Health Science Centre;

${ }^{7}$ Arthritis Research UK Centre for Genetics and Genomics, the University of Manchester, Manchester, United Kingdom

Background: Identifying predictors for early clinically inactive disease (CID) would allow stratified treatment decisions at diagnosis, minimising the burden of unnecessary therapies. JIA core outcome variables (COVs) are routinely collected and would therefore be convenient predictors. However, different groups of children are identified by current CID definitions (Wallace's preliminary criteria vs. clinical Juvenile Arthritis Disease Activity Score in 10 joints (cJADAS10)) and predictors may differ depending on which CID outcome is applied.

Objectives: To assess whether baseline COVs predict achievement of CID according to Wallace's preliminary criteria or the cJADAS10 cut-off in patients with JIA.

Methods: Children and young people enrolled to the Childhood Arthritis Prospective Study (CAPS), a UK multicentre inception cohort, before January 2011, were selected if diagnosed with oligoarticular, RF-negative or RF-positive polyarticular JIA.

At one year following initial presentation to paediatric rheumatology, children were classified as i) CID according to Wallace's preliminary criteria and ii) CID according to CJADAS10. Baseline COVs (active joint count, limited join count, physician's global, parental global, functional ability (Childhood Health Assessment Questionnaire (CHAQ)) and ESR) were tested for predictive ability for these outcomes using univariate and forced-entry multivariate logistic regressions, adjusting for age and symptom duration at initial presentation, gender and ILAR subtype. Multiple imputation accounted for missing data.

Results: Of 829 children included, $70 \%$ were female and the majority had oligoarticular JIA (68\%). At one year, $28 \%$ had achieved CID according to Wallace's preliminary criteria and $38 \%$ according to the cJADAS $10(21 \%$ CID on both).

In univariate analyses, increased baseline $\mathrm{CHAQ}$ and physician's global assessment score predicted lower odds of achieving both CID states. In addition, increased active joints (OR: 0.97, 95\% Cl0.94, 0.99), and patient/parent global assessment scores (OR: $0.87,95 \% \mathrm{Cl} 0.81,0.93$ ) predicted lower odds of CID on the cJADAS10 only. In multivariate analyses, one increased CHAQ point at baseline independently predicted $28 \%$ lower odds of CID on the CJADAS 10 $(95 \% \mathrm{CI} 0.53,0.98)$. However, no baseline COVs predicted CID on Wallace's preliminary criteria. No demographic variables were significantly predictive in any model.

Conclusions: There were different predictors for CID on the cJADAS10 vs. Wallace's preliminary criteria. Children with poor functional ability at initial presentation are less likely to achieve CID on the cJADAS10. These children could be targeted with more aggressive treatment strategies to better control their disease.

Disclosure of Interest: None declared

DOI: 10.1136/annrheumdis-2017-eular.3738

\section{THU0534 BASELINE CHARACTERISTICS AND DESCRIPTIVE SAFETY DATA OF INTRAVENOUS ABATACEPT-TREATED PATIENTS WITH JUVENILE IDIOPATHIC ARTHRITIS IN A US HEALTHCARE CLAIMS DATABASE}

T. Simon $^{1}$, S. Singhal ${ }^{2}$, N. Ray ${ }^{2}$, Z. Guo ${ }^{1} .{ }^{1}$ Bristol-Myers Squibb, Princeton, United States; ${ }^{2}$ Mu Sigma, Bangalore, India

Background: Abatacept (ABA), the first selective co-stimulation modulator approved and used for the treatment of moderate-to-severe polyarticular juvenile idiopathic arthritis (JIA), has a mechanism of action that is different from other biologic (b)DMARDs.

Objectives: To describe the baseline $(B L)$ characteristics and validated safety outcomes of patients ( $p t s)$ with a diagnosis of JIA in a US healthcare claims database treated with IV ABA and those with a minimum of 12 months (M) of ABA treatment.

Methods: Pts aged $<18$ years (yrs), diagnosed with JIA and in the Truven Health MarketScan ${ }^{\circledR}$ database between 1 Jan 2006 and 30 Sep 2014 were eligible for inclusion. Pts were required to have $\geq 180$ days (d) of continuous health plan enrolment prior to a diagnosis of JIA based on two International Classification of Diseases, Ninth Revision, Clinical Modification codes (714.3x) within $90 \mathrm{~d}$. BL characteristics and prior bDMARD use were analysed for two IV ABA-treated groups: a total cohort and a subgroup of pts with $\geq 12 \mathrm{M}$ of treatment. Three categories evaluated prior bDMARD use claims: biologic holiday (other bDMARD in history but not within $180 \mathrm{~d}$ of $\mathrm{BL}$ ), biologic switcher (other bDMARD within $180 \mathrm{~d}$ of $\mathrm{BL}$ ) and true initiator (no history of other bDMARD). Incidence rates (IR; number of events/person yrs [p-y] of exposure) per 100 p-y of exposure with $95 \%$ $\mathrm{Cl}$ were calcuated for infections, malignancies and uveitis.

Results: A total of 238 IV ABA-treated pts were identified with 89 pts having $\geq 12 \mathrm{M}$ of ABA treatment. The mean (SD) follow-up duration was 1.73 (1.28) yrs for the total IV ABA cohort and 2.28 (1.03) yrs for the $\geq 12-\mathrm{M}$ subgroup. Most pts were female, and mean age was 12.4 yrs. Overall, the total IV ABA cohort was more likely to have a claim in the BL period for asthma and cardiovascular disease versus the $\geq 12-\mathrm{M}$ subgroup; the $\geq 12-\mathrm{M}$ subgroup was more likely to have uveitis (Table). The most frequent other bDMARD claim for the total IV ABA-treated pts in the biologic holiday group was etanercept (41.9\%), and adalimumab for

\begin{tabular}{lcc}
\hline BL Characteristics & Total IV ABA cohort $(\mathrm{n}=238)$ & IV ABA $\geq 12-\mathrm{M}$ subgroup $(\mathrm{n}=89)$ \\
\hline Female, $\mathrm{n}(\%)$ & $195(81.9)$ & $78(87.6)$ \\
Age, mean (SD) & $12.4(3.2)$ & $12.4(3.2)$ \\
Uveitis, $\mathrm{n}(\%)$ & $22(9.2)$ & $11(12.4)$ \\
Asthma, $\mathrm{n}(\%)$ & $21(8.8)$ & $6(6.7)$ \\
Cardiovascular disease, $\mathrm{n}(\%)$ & $18(7.6)$ & $6(6.7)$ \\
bDMARDs, $\mathrm{n}(\%)$ & $84(35.3)$ & $30(33.7)$ \\
Non-biologic DMARDs, $\mathrm{n}(\%)$ & $105(44.1)$ & $37(41.6)$ \\
Inpatient visits, mean (SD) & $0.2(0.8)$ & $0.1(0.3)$ \\
Outpatient visits, mean (SD) & $11.6(10.7)$ & $10.0(9.8)$ \\
\hline
\end{tabular}

Abstract THU0532 - Table 2

\begin{tabular}{|c|c|c|c|c|}
\hline Treatments & I: Anterior uveitis (42) & II: Intermediate uveitis (16) & III: Posterior uveitis (7) & IV: Panuveitis (9) \\
\hline High dose systemic steroid & $45 \%(19)$ & $80 \%(12)$ & $71 \%(5)$ & $100 \%(9)$ \\
\hline Synth DMARDs & 66\% (27) (MTX-26, AZA-2) & 60\% (9) (MTX, AZA) & $71 \%$ (5) (MTX, AZA) & 78\% (7) (MTX, AZA, COL) \\
\hline SynthDMARDs + Biologics & $34 \%(14)(I F X-4, A D A-10)$ & $33 \%(5)($ IFX-1, TCZ-1, IFN-4) & $43 \%$ (3) (IFX-2, IFN-1) & $33 \%(3)(I F X-3)$ \\
\hline Surgery & $18 \%$ (7) (cataracts -5 , glaucoma -1 , keratopathy -1 ) & $20 \%$ (3) (cataracts -3 , vitrectomy -1 ) & 0 & $\begin{array}{c}63 \% \text { (5) (cataracts }-2 \text {, glaucoma }-1, \\
\text { vitrectomy }-1 \text {, antiangio. inj.-1) }\end{array}$ \\
\hline
\end{tabular}

